\title{
Correction to: NiCad: A Modern Clone Entector
}

\author{
Manishankar Mondal, Chanchal K. Roy, and James R. Cordy
}

\section{Correction to:}

Chapter "NiCad: A Modern Clone Detector" in: K. Inoue and C. K. Roy (eds.),

Code Clone Analysis, https://doi.org/10.1007/978-981-16-1927-4_3

In the original version of the book, the following belated correction has been incorporated: In chapter "NiCad: A Modern Clone Detector", the author "J. R. Cordy's" affiliation has been changed from "Queen's University, Belfast, Northern Ireland" to "Queen's University, Kingston, Canada".

The correction chapter and the book have been updated with the change. 